\title{
Recent Development of Photo-Cross- Linkers as Tools for Biomedical Research
}

\author{
Yulin Tian and Qing Lin*
}

\begin{abstract}
Photo-cross-linkers are invaluable tools for identifying drug targets and off-targets as well as probing the binding sites in medicinal chemistry and chemical biology. In this review, we summarize recent development of the ligand-based and genetically encoded photo-cross-linkers and their use in biological system. In particular, we highlight our discovery of 2-aryl-5-carboxytetrazole (ACT) as a novel class of photo-cross-linkers and their successful applications in drug target identification as well as identifying transient protein-protein interaction complexes in mammalian cells.
\end{abstract}

Keywords: Genetic encoding · Photo-affinity probe · Photo-cross-linker · Protein-protein interaction · Target identification

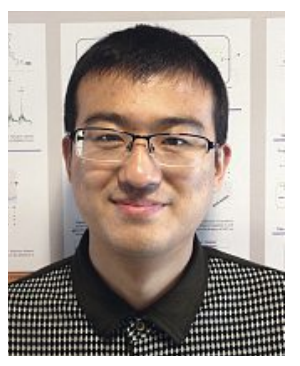

Dr. Yulin Tian was born in Hebei, China. He obtained his BS in pharmacy from Shenyang Pharmaceutical University in 2010, and his $\mathrm{PhD}$ in medicinal chemistry from Institute of Materia Medica, Peking Union Medical College in 2015 under the direction of Professor Dali Yin. He then worked in Professor Qing Lin's lab at State University of New York at Buffalo as a postdoctoral researcher. His research focuses on design of the genetically encoded photo-crosslinkers, stapled oxyntomodulin analogs as GLP-1R/GCGR dual agonists, and the bioorthogonal chemical probes for the study of ligand-induced class B GPCR activation.
${ }^{*}$ Correspondence: Prof. Q. Lin 679 Natural Science Complex Department of Chemistry

State University of New York at Buffalo

Buffalo, New York 14260, USA

E-mail: qinglin@buffalo.edu

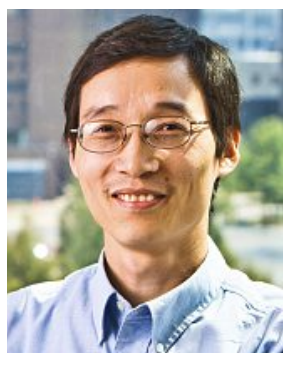

Professor Qing Lin received his $\mathrm{PhD}$ in Organic Chemistry from Yale University in 2000 under the direction of Professor Andrew Hamilton. After postdoctoral work in Professor Peter Schultz's lab at Scripps Research Institute and a brief stay in industry, Dr. Lin joined the faculty of State University of New York at Buffalo in 2005 where he is now Professor of Chemistry. His research group is interested in harnessing chemical reactivity for studies of protein structure and function in living systems.

\section{Introduction}

Photo-cross-linking offers a powerful technique to study transient protein-ligand and protein-protein interactions in complex proteome. ${ }^{[1]}$ Upon photoirradiation, the highly reactive species are generated from photo-cross-linkers, which then react with the proximal residues on target proteins to form the covalent bond. Because photo-cross-linking enables covalent capture of transient interaction partners with a high spatiotemporal resolution in live cells, photo-cross-linkers have emerged as a critical tool for drug target identification, discovery of off-targets, activity-based protein profiling (ABPP), and structural elucidation of the binding sites within protein targets. ${ }^{[2]}$

The classical photo-cross-linkers include benzophenone (BP), phenyl azide (PA), and diazirine (DA), wherein the reactive intermediates after irradiation with lights of varying wavelength include di- radical, nitrene, and carbene, respectively. These highly reactive intermediates react with neighboring $\mathrm{C}-\mathrm{H}$ or heteroatom- $\mathrm{H}$ bonds to form the photo-cross-linked adducts (Scheme 1). Recently, our group serendipitously discovered 2-aryl-5-carboxytetrazole (ACT) as a new and potentially superior photo-cross-linker because of its higher photo-cross-linking efficiency and specificity. ${ }^{[3]}$ Upon UV irradiation, ACT undergoes tetrazole ring rupture to generate the electrophilic carboxynitrile imine, which then reacts with a proximal nucleophile such as thiol, alcohol, carboxyl acid and amine via the nucleophilic addition (Scheme 1). ${ }^{[4]}$

\section{Classical Photo-Cross-Linkers}

\subsection{Photo-Affinity Probes}

Photo-affinity probes are reagents that conjugate the photo-cross-linkers with small-molecule ligands with a goal to study the ligand-protein interactions. The design of photo-affinity probes has attracted intense interest in drug development because it greatly facilitates the use of activity-based protein profiling (ABPP) for drug target identification and structural characterization. ${ }^{[5]}$ The photo-affinity probes are usually comprised of three parts: (i) ligand warhead for target engagement; (ii) photocross-linker (BP, PA or DA) for covalent capture; and (iii) bioorthogonal tag for fluorescent detection or target enrichment. In a general workflow, cells or cell lysates are treated with photo-affinity probes for a certain time followed by photoirradiation with specific wavelength of light to photocross-link with potential targets. The bioorthogonal reporter tagged proteins in cell lysates are allowed to react with biotin or a fluorescent probe for affinity purifi- 
Scheme 1. Photocross-linkers and their respective reactive intermediates and photo-cross-linking products.

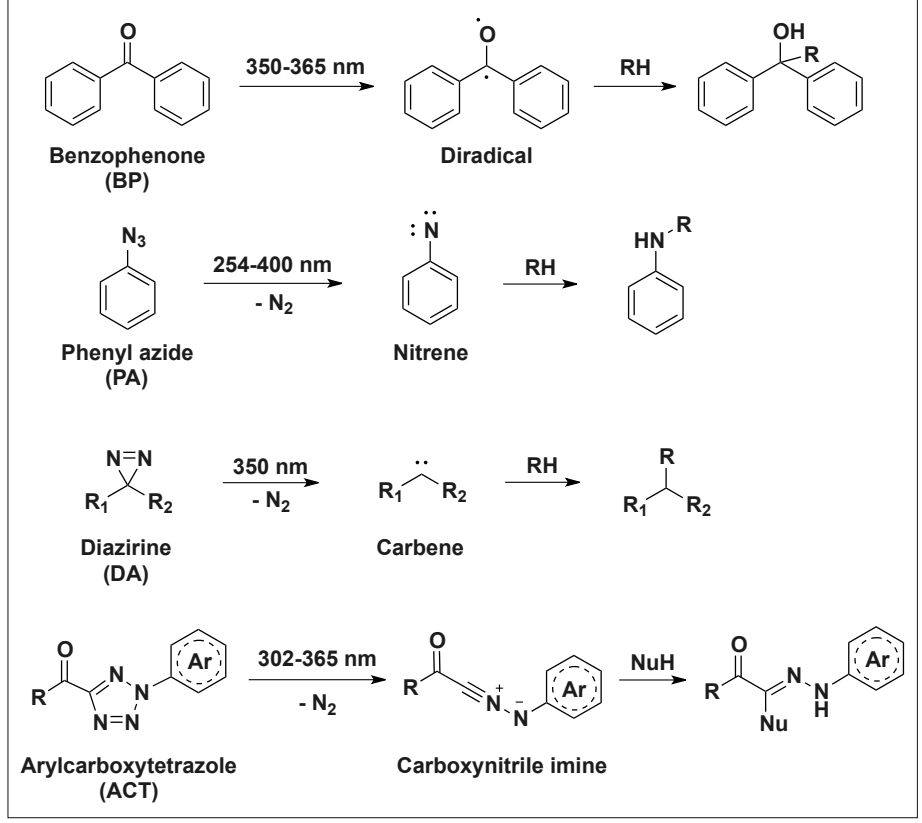

cation or fluorescence-based visualization on SDS-PAGE gel. For target identification, the labeled proteins are excised from the gel and their identities are determined by LC-MS/MS after digestion with trypsin. For example, Cravatt and co-workers reported the design of an activity-based photoaffinity probe, HxBP-Rh (Fig. 1), for metalloproteases (MPs) by conjugating a zinc-chelating hydroxamate-based ligand to benzophenone. ${ }^{[6]}$ By profiling the activity of MPs in cell and tissue samples, they were able to identify the highly upregulated MPs in invasive cancer cells.

Since targets of many bioactive natural products have not been identified, Kotake and co-workers designed a photo-affinity probe by conjugating a trifluoromethyl phenyl diazirine photo-cross-linker with the antitumor natural product pladienolide (Fig. 1). ${ }^{[7]}$ Using a fluorescent probe and a radiolabel, a spliceosome associated pro- tein, SAP130, was identified as the target of pladienolide, suggesting that the mRNA splicing machinery may be a viable antitumor drug target. Sieber and co-workers designed the benzophenone-based photoaffinity probe of glycopeptide antibiotic vancomycin (Fig. 1) for identification of potential vancomycin binders in pathogenic bacteria. ${ }^{[8]}$ Proteomic profiling revealed two previously unknown vancomycin targets: a bifunctional autolysin and a peptide $\mathrm{ABC}$ transporter; both targets were further validated through recombinant expression and labeling.

For some promising clinical candidates, their molecular targets remain to be elucidated. LW6 was known to inhibit mulation and its transcriptional activity. ${ }^{[9]}$ To identify the target, Lee et al. designed a diazirine-based photo-affinity probe of LW6 based on the prior SAR studies (Fig. hypoxia-inducible factor (HIF-1 $\alpha$ ) accu-
1).[10] Chemical proteomic analysis uncovered a mitochondrial enzyme, malate dehydrogenase 2 (MDH2), as the target, which was further validated by subsequent in vitro studies. The Park group discovered an isoxazole compound P29C06 as an enhancer of cellular glucose uptake after screening a 3000-compound library. ${ }^{[1]}$ Target identification was carried out using a phenyl azide containing photo-affinity probe, P29C06-Az (Fig. 1). PPAR- $\gamma$, a protein associated with insulin sensitization, was identified as a potential target. Further optimization of the initial hit led to a lead compound with 4000-fold higher activity.

In addition to target identification, photoaffinity probes can also be used to elucidate the ligand binding sites in target proteins. For example, the inhibitors of $\mathrm{Na}^{+} / \mathrm{H}^{+}$antiporters, amilorides, are known to inhibit the NADH-quinone oxidoreductase (complex I). ${ }^{[12]}$ To elucidate the binding sites, Murai et al. introduced the phenyl azide to obtain the photo-affinity probe PRA1 (Fig. 1), and performed the photocross-linking reaction with bovine heart submitochondrial particles. ${ }^{[13]}$ Proteomic analysis revealed that PRA1 specifically labels accessory subunit B14.5a and the N-terminal region of a $49-\mathrm{kDa}$ core subunit, indicating that amilorides inhibit complex I by occupying the quinone binding pocket.

The protein-lipid interaction is an essential metabolic process that mediates cellular signaling and function. ${ }^{[14]}$ The Lei group introduced a diazirine to the aliphatic chain of the glycolipid, monogalactosyldiacylglycerol (MGDG), to derive a photoaffinity probe (Fig. 1) for mechanistic studies of the glycolipid-protein interaction. ${ }^{[15]}$ After proteomic labeling and subsequent pull-down and MS analysis, toll-like receptor 4 (TLR4) was identified as the target of MGDG. Further biochemi-

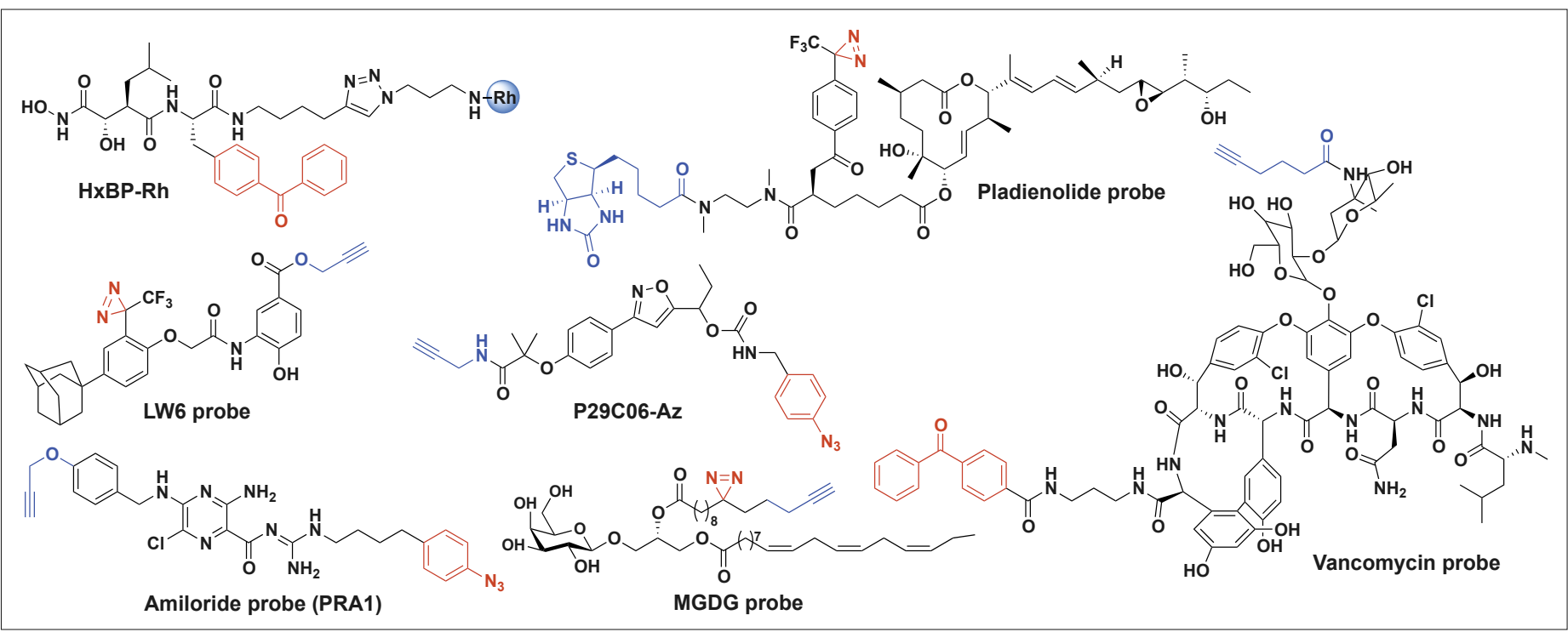

Fig. 1. Structures of photo-affinity probes. 
cal studies suggested that the anti-inflammatory activity of MGDG was a result of direct antagonism of TLR4.

\subsection{Genetically Encoded Photo- \\ Cross-Linkers}

Aside from their use in photo-affinity probes, photo-cross-linkers have also been introduced into proteins via genetic code expansion to study transient protein-protein interactions (PPIs) in native cellular environments. ${ }^{[16]}$ Pioneered by Schultz and others, the genetic code expansion technology allows site-specific incorporation of unique chemical functionalities as part of unnatural amino acids (UAAs) into a protein of interest in response to amber codon suppression using engineered aminoacyltRNA synthetase/tRNA pairs. ${ }^{[17]}$

In 1986, Kauer et al. reported the incorporation of a benzophenone-containing amino acid, $p$-benzoyl-L-phenylalanine $(\mathrm{BzF})$ into a peptide using solid-phase peptide synthesis (Fig. 2a). [18] From 2002 to 2007, Schultz and coworkers reported site-specific incorpora-

tion of three phenylalanine derivatives, $\mathrm{BzF},[19] \quad p$-azido-L-phenylalanine (azF) [20] and 4'-[3-(trifluoromethyl)-3H-diazirin-3-yl]-L-phenylalanine (tmdF), ${ }^{[21]}$ into proteins in Escherichia coli using the evolved tyrosyl-tRNA synthetase (TyrRS) mutants (Fig. 2a). These UAAs have a cross-linking radius around 6 8 $\AA$ suitable for mapping short-range PPIs. For example, BzF and azF were incorporated into $\mathrm{F} 52$ position at the dimer interface of Schistosoma japonica glutathione $S$-transferase ( $s j \mathrm{GST}$ ), and enabled efficient photo-cross-linking of GST dimer in vitro. Yokoyama and co-workers extended the utility of BzF to mammalian systems by demonstrating successful capture of epidermal growth factor receptor (EGFR) using the BzF-encoded Src homology 2 (SH2) mutant of the adapter protein Grb2. [22] The same group also developed tmdF, a diazirine containing phenylalanine derivative (Fig. 2a), and used it to identify several novel Grb2 interacting proteins including nuclear ribonucleoproteins $\mathrm{F}$, $\mathrm{H} 1$, and $\mathrm{H} 2 .{ }^{[23]}$

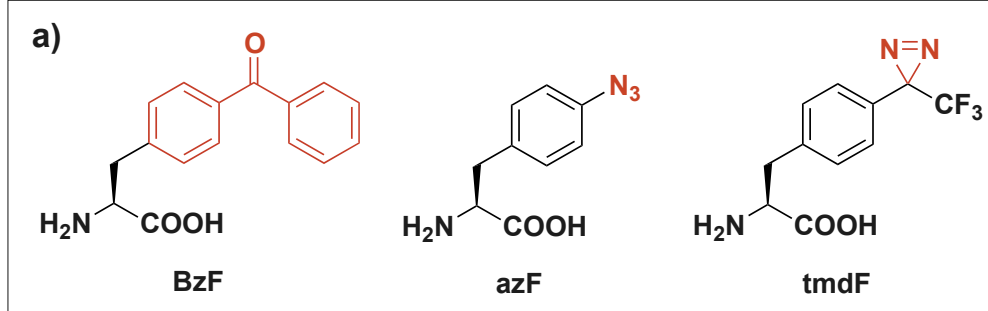

b)
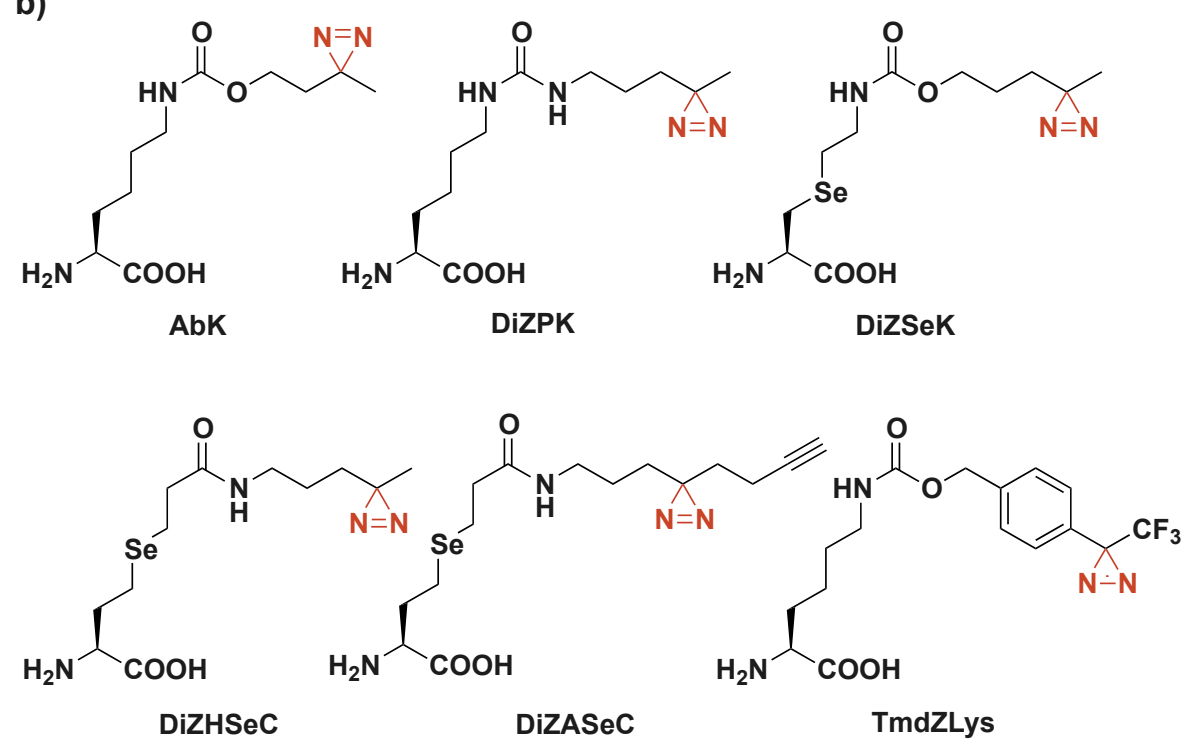

c)
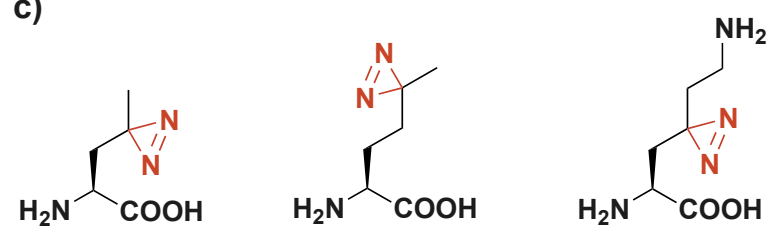

Photo-Leu

Photo-Met

Photo-Lys
The photo-cross-linking amino acids $\mathrm{BzF}$ and azF also proved to be valuable in elucidating the ligand-binding sites. For example, the Sakmar group used BzF and azF to map the potential ligand binding sites in several G-protein coupled receptors (GPCRs), ${ }^{[24]}$ including $\mathrm{CXC}$ chemokine receptor 4 (CXCR4), ${ }^{[25]} \mathrm{CC}$ chemokine receptor 5 (CCR5), ${ }^{[26]}$ and glucagonlike peptide- 1 receptor (GLP-1R). ${ }^{[27]}$ After stimulation with the fluorescein or $\left[{ }^{3} \mathrm{H}\right]-$ labeled ligands, the ligand-binding sites were mapped by analyzing UV-dependent photo-cross-linking with the labeled ligands. Similarly, Wang and co-workers employed azF to map the interaction interface between corticotropin releasing factor receptor type 1 (CRF1R) and its ligand, urocortin-I, yielding insights into the receptor conformational transitions during ligand binding. ${ }^{[28]}$ The Strømgaard group employed $\mathrm{BzF}$ and azF to identify the binding sites of four different antidepressant drugs (imipramine, vortioxetine, escitalopram and paroxetine) in human serotonin transporter (hSERT), yielding the structural details about the clinically relevant antidepressant target. [29]

Another application of $\mathrm{BzF}$ and azF is to identify new PPI targets. To this end, Fierke and co-workers incorporated $\mathrm{BzF}$ at the active site of histone deacetylase 8 (HDAC8) with a goal to capture its substrates. ${ }^{[30]}$ The BzF-encoded recombinant HDAC8 was incubated with the HEK293T cell lysate and the mixture was irradiated with UV light. LC-MS analysis uncovered more than 100 active site engaged protein substrates. Mapp and co-workers employed BzF to identify the binding partners of transcriptional activator Gal4. [31] Photocross-linking with a series of BzF-encoded Gal4 mutants identified a key masking protein Gal80 and the corresponding binding interface. Furthermore, by combining genetic encoding of $\mathrm{BzF}$ with label-free shotgun LC-MS, they discovered that AMPK kinase complex interacts with Gal4 and VP16 via core enzymatic subunit Snf1 and exchangeable subunit Gal83.[32]

Besides TyrRS, pyrrolysyl-tRNA synthetase (PylRS) and its variants have shown tremendous versatility in charging the lysine derivatives into proteins in bacteria, yeast and mammalian cells. ${ }^{[33]} \mathrm{A}$ number of aliphatic diazirine-containing lysine derivatives were reported as substrates of PylRS mutants. For example, the Deiters and Schultz groups reported the design of 3'-azibutyl- $N$-carbamoyllysine (AbK; Fig. 2b) as a genetically encodable photo-cross-linker with crosslinking radius of $12 \AA$, allowing efficient photo-cross-linking of GST dimer. [34] Because of its small size and flexible chain, $\mathrm{AbK}$ is expected to have minimum perturbation to protein structure compared

Fig. 2. Genetically encoded photo-cross-linking amino acids. 
to $\mathrm{BzF}$ and azF. Chen and co-workers developed (3-(3-methyl-3H-diazirine-3-yl)propaminocarbonyl- $N^{\varepsilon}$-L-lysine (DiZPK; Fig. 2b) with cross-linking radius of 14 $\AA$, and used it to identify the client proteins of HdeA, an acid resistance chaperone in bacteria. ${ }^{[35]}$ Photo-cross-linking followed by mass spectrometry produced a list of HdeA client proteins including DegP and SurA that assist the HdeAmediated substrate refolding in E. coli. In addition, the same group developed three selenium-based cleavable DiZPK analogs, DiZSeK, DiZHSeC and DiZASeC (Fig. 2b) for separating the prey-bait complexes after oxidative cleavage of the Se-C bond.[36] DiZSeK was generated by replacing $\gamma$-carbon with selenium in DiZPK, which allowed covalent trapping of the prey proteins in live cells and subsequent separation of the bait from the prey protein upon $\mathrm{H}_{2} \mathrm{O}_{2}$ treatment.[37] To map PPI interface, the Chen group integrated a transferable, MS-identifiable label (MSlabel) into DiZPK to derive DiZHSeC, which generates a stable MS-label on the captured client proteins after photo-crosslinking and subsequent cleavage. ${ }^{[38]}$ This strategy improved specificity, fidelity and robustness during target identification and enabled identification of 28 new HdeA client proteins and mapping of the HdeADegP interaction interface. More recently, a bioorthogonal alkyne handle was introduced by the Chen group into DiZHSeC to produce a multifunctional photo-crosslinker, DiZASeC, which enabled the enrichment of transient and low-abundance prey proteins after in situ photo-crosslinking and subsequent cleavage. ${ }^{[39]}$ Using stable isotope labeling and MS analysis, six new low-abundance substrates of DegP were uncovered. Separately, Yanagisawa et al. developed a trifluoromethyl phenyl diazirine-containing photo-cross-linker, TmdZLys (Fig. 2b), which represents a 'wide-range' photo-cross-linker with cross-linking radius of $15 \AA .{ }^{[40]}$ TmdZLys not only captures the interaction partners over a long distance but also reacts with those in close proximity, offering a potentially more efficient tool for global profiling of unknown substrates.

The structural similarity between the photo-cross-linker-bearing UAAs and naturally occurring methionine and leucine allows native mammalian translational machinery to charge these UAAs into proteins in a residue-specific manner. To this end, the Thiele group reported metabolic incorporation of two diazirine-containing amino acids, photo-methionine (PhotoMet) and photo-leucine (Photo-Leu; Fig. 2c) into proteomes in mammalian cells. ${ }^{[41]}$ Through photo-cross-linking and mass spectrometry, the previously unknown interactions of progesterone receptor membrane component 1 (PGRMC1) with Insig-1 and SCAP were identified. Since the post-translationally modified (PTM) lysines are crucial for epigenetics, $\mathrm{Li}$ and co-workers introduced the diazirine group onto the lysine side chain to obtain PhotoLys (Fig. 2c), which can be readily incorporated into proteins through mammalian translation machinery. ${ }^{[42]}$ Photo-Lys and the PTM-containing photo-lysine were then used to identify proteins that recognize lysine PTMs, including the 'readers' and 'erasers' of histone modification.

\section{ACT-based Photo-Cross-Linkers}

3.1 ACT-based Photoaffinity Probes

In 2016, our group reported a new class of photo-cross-linkers, 2-aryl-5-carboxytetrazole (ACT), that upon conjuga- tion with a drug allows identification of the drug targets via a unique mechanism. ${ }^{[3]}$ The photoinduced carboxy-nitrile imine generated from ACT is capable of undergoing rapid reaction with a proximal nucleophile such as glutamic acid to form the covalent adduct. Because of its unique mechanism, ACT has shown greater cross-linking specificity than the traditional photo-cross-linkers such as BP, PA and DA that work through nonspecific $\mathrm{C}-\mathrm{H} / \mathrm{X}-\mathrm{H}$ bond insertion. For example, the ACT moiety was attached to dasatinib and JQ-1 (a BRD4 inhibitor) to afford two series of photo-affinity probes with varying length of linkers (Fig. 3a). The in vitro binding assay indicated that ACT attachment did not significantly alter the binding affinity and specificity. In LC-MS-based photo-cross-linking studies, the ACT-based probes showed higher photo-cross-linking yields (60-95\%) with their protein targets in vitro than the DA and BP-based ones along with reduced backgrounds. The cross-linking site of ACT-based dasatinib probe on BTK was mapped to Glu-488 based on the LC-MS/ MS analysis. Glu-488 is located near the ligand binding pocket, with the distance between the C5 of ACT and the carboxylate oxygen of Glu-488 to be $6.9 \AA$ based on a docking model. The proposed mechanism of the ACT-mediated photo-crosslinking involves nucleophilic addition of Glu-488 carboxylate to the carboxy-nitrile imine followed by 1,4-acyl shift. ${ }^{[3]}$

ACT was then used for in situ target identification in intact K562 cells. The known targets of dasatinib, BTK, Src, and Csk, as well as the targets of JQ-1, BRD4, were successfully captured by their respective photo-affinity probes. In addition, ingel digestion followed by LC-MS/MS analysis produced lists of potential off-targets.

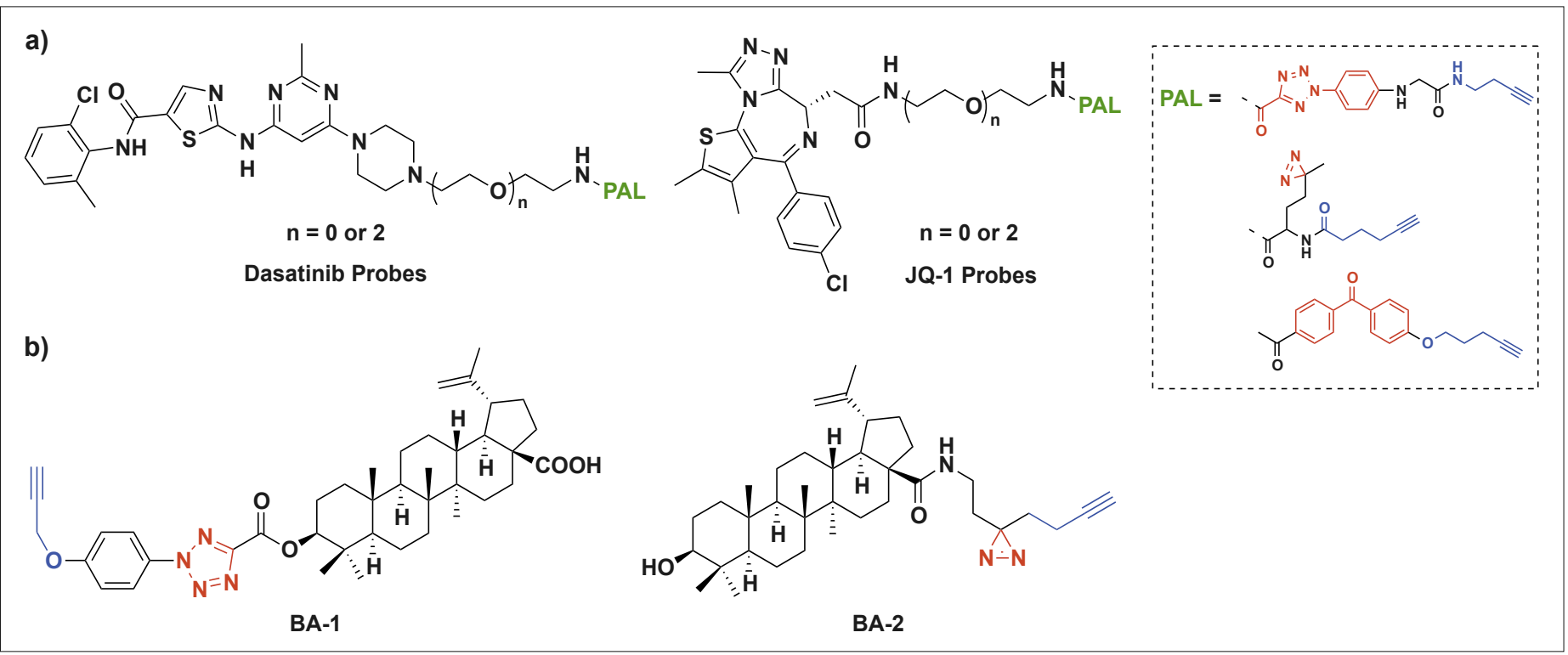

Fig. 3. Structures of ACT-based photo-affinity probes. 
Compared with DA and BP, the main advantage of ACT lies in its unique mechanism, which can lead to reduced background reactions with nonspecific targets as well as more facile mapping of the ligand-binding site. A potential drawback of ACT is that a suitable nucleophile needs to be in place near the ligand-binding site for the targets to be captured, which may lead to false negative when the necessary nucleophile is absent. However, this limitation can in principle be overcome by increasing the linker length between ACT and the ligand to allow the survey of a larger area surrounding the ligand-binding pocket.

After our initial report, Guo et al. used ACT as part of their photo-affinity labels to identify potential cellular targets of the natural product, betulinic acid (BA), in cancer cells. ${ }^{[43]}$ The ACT and DA-containing photo-affinity probes, BA-1 and BA-2, were used in proteomic profiling and imaging studies (Fig. 3b). Interestingly, after in situ photo-crosslinking, pull-down, and LC-MS/MS analysis, different protein targets were identified using these two probes ( 9 proteins for BA-1 and 13 proteins for BA-2), which can be attributed to the use of different linkage sites on BA. Among the potential targets, the BA-1-identified proteins have not been reported previously, highlighting the advantage of ACT's unique photo-cross-linking mechanism. Two disease-related and high-confidence proteins (PFKFB1 and C3) were further validated in subsequent affinity pull-down followed by western blot analysis.

\subsection{ACT-based Genetically Encoded Photo-Cross-Linkers}

Besides ligand-directed photo-crosslinking, we also demonstrated that the ACT-modified lysine derivatives (ACTK; Fig. 4) can serve as a new class of genetically encoded photo-cross-linkers for mapping transient protein-protein interactions in mammalian cells. Initially, five ACTK derivatives were readily prepared through acylation of the lysine $\varepsilon$-amino group with an activated ACT carboxyl ester. ${ }^{[44]}$ An $M$. mazei PylRS mutant carrying Y306V/L309A/C348F/Y384F mutations, referred to as mPyTKRS, was identified from the PylRS library. Using the orthogonal mPyTKRS/tRNA ${ }_{\text {CUA }}$ pair, mPyTK was readily incorporated into sfGFP in E. coli, along with four related ACTK analogs including PyTK, FTK, TTK and PhTK (Fig. 4).

Then the efficiency of ACTK in photocross-linking of the GST dimer was assessed. In brief, mPyTK was placed at Glu-52 position at the dimer interface, and indeed time-dependent photo-cross-linking of the GST dimer was observed both in vitro and inside E. coli cells. Compared to the diazirine-containing AbK amino acid (structure shown in Fig. 2b), mPyTK exhibited significantly higher photo-cross-linking efficiency when both were placed at Glu-52 position. The photo-cross-linking site in the mPyTK-encoded GST dimer was mapped to Glu-92 via alanine scanning, confirming proximity-dependent reactivity as Glu- 92 is the closest nucleophilic residue. A proposed photo-cross-linking mechanism involves nucleophilic addition of the Glu-92 carboxylate to the photo-generated carboxynitrile imine followed by acyl shift, which was supported by identification of the rearranged cross-linked product during the mass spectrometry analysis. When mPyTK was introduced into the suitable positions in Grb2, an adaptor protein mediating the receptor tyrosine kinase signaling, the timeand stimulus-dependent covalent capture of its interaction partner EGFR was observed in live mammalian cells.

To improve expression yield of the ACTK-encoded proteins, three additional ACTK derivatives (mPyTXK) were synthesized in which the $\gamma$-carbon was replaced with oxygen, sulfur and selenium, respectively (Fig. 4). ${ }^{[45]}$ Compared to mPyTK, the heteroatom-substituted ACTK derivatives (mPyTOK, mPyTSK, mPyTSeK) are more hydrophilic with lower $\operatorname{cLog} P$ values. Indeed, mPyTXK afforded higher expression yields when incorporated into position-204 of sfGFP using the mPyTKRS/tRNAPyl ${ }_{\text {CUA }}$ pair. Moreover, mPyTXK was site-specifically incorporated into the mCherry-TAG-EGFP-HA fusion protein based on fluorescence microscopy, confirming the polyspecificity of mPyTKRS in charging ACTK analogs in mammalian cells. Two of the mPyTK analogues, mPyTSK and mPyTSeK, enabled site-selective photo-cross-linking of the
GST dimer in vitro at an efficiency comparable to mPyTK.

Since the $\mathrm{C}^{\gamma}-\mathrm{Se}$ bond can be cleaved under mild oxidation conditions, mPyTSeK was tested as a cleavable photo-crosslinker to separate the cross-linked adducts. This was demonstrated through the complete conversion of the GST dimer back to the monomer after treating the photo-crosslinked mPyTSeK-encoded GST dimer with $\mathrm{H}_{2} \mathrm{O}_{2}$. Furthermore, the mPyTSeK-encoded Grb2 protein was able to photo-capture EGFR in mammalian cell lysates, which was subsequently released after treatment with $\mathrm{H}_{2} \mathrm{O}_{2}$. These results verified the unique capability of mPySeK as a cleavable photocross-linker, which may allow rapid mapping of the protein-protein interaction complexes in mammalian cells.

\section{Conclusion and Outlook}

In conjunction with the use of bioorthogonal tags, the applications of the photo-cross-linkers in biomedical research have drawn renewed interest in recent years. A wide range of photo-affinity probes and the genetically encoded photo-cross-linkers have been designed and used to study significant problems in medicinal chemistry and chemical biology, including proteomic profiling, target and off-target identification, binding site determination, and mapping of transient protein-protein interactions and their interfaces. Because of its unique mechanism, the newly discovered ACT-based photo-cross-linkers have shown several advantages over the traditional photocross-linkers, including higher photocross-linking yield and greater photocross-linking specificity for proximal nucleophilic residues in target proteins. However, because each photo-crosslinker possesses discrete physicochemical and photo-cross-linking properties, it might be advantageous to combine these photo-cross-linkers in the design of the photoaffinity probes and the genetically encoded analogs for target identification and PPI mapping, respectively, in biological systems. By comparing and contrasting the target lists derived from different photo-cross-linkers, high-confidence targets can be obtained along with drastically reduced nonspecific binding. ${ }^{[46]}$

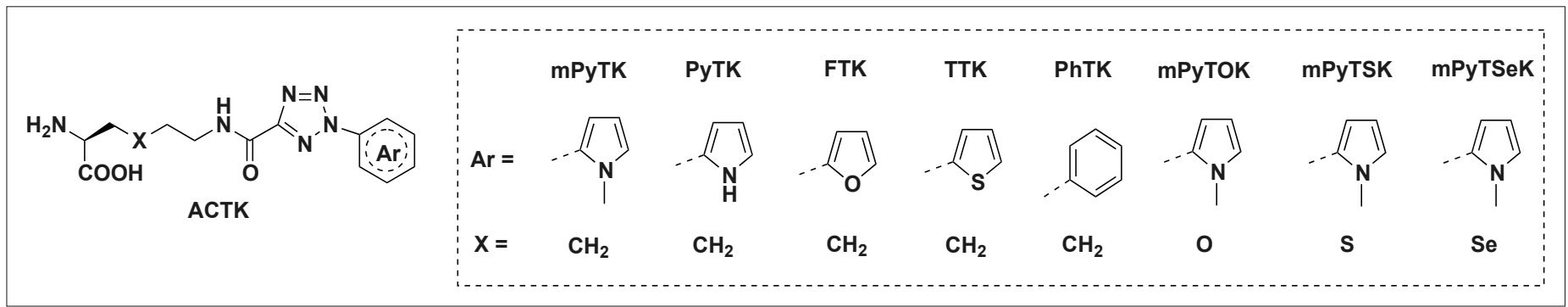

Fig. 4. Structures of ACT-based genetically encoded photo-cross-linkers. 


\section{Acknowledgements}

We gratefully acknowledge the $\mathrm{NIH}$ (GM085092) and AbbVie for financial support of our work in this area.

Received: May 18, 2018

[1] a) Y. Tanaka, M. R. Bond, J. J. Kohler, Mol. Biosyst. 2008, 4, 473; b) D. P. Murale, S. C. Hong, M. M. Haque, J. S. Lee, Proteome. Sci. 2016, 15, 14; c) G. W. Preston, A. J. Wilson, Chem. Soc. Rev. 2013, 42, 3289.

[2] a) D. Robinette, N. Neamati, K. B. Tomer, C. H Borchers, Expert. Rev. Proteomic. 2006, 3, 399 ; b) N. D. Pham, R. B. Parker, J. J. Kohler, Curr Opin. Chem. Biol. 2013, 17, 90; c) E. Smith, I Collins, Future. Med. Chem. 2015, 7, 159; d) D J. Lapinsky, D. S. Johnson, Future. Med. Chem. 2015, 7, 2143; e) M. H. Wright, S. A. Sieber, Nat. Prod. Rep. 2016, 33, 681.

[3] A. Herner, J. Marjanovic, T. M. Lewandowski, V. Marin, M. Patterson, L. Miesbauer, D. Ready, J. Williams, A. Vasudevan, Q. Lin, J. Am. Chem Soc. 2016, 138, 14609.

[4] R. Huisgen, J. Sauer, M. Seidel, Chem. Ber. 1961, 94, 2503

[5] J. Park, M. Koh, S. B. Park, Mol. Biosyst. 2013, $9,544$.

[6] A. Saghatelian, N. Jessani, A. Joseph, M. Humphrey, B. F. Cravatt, Proc. Natl. Acad. Sci. USA 2004, 101, 10000.

[7] Y. Kotake, K. Sagane, T. Owa, Y. MimoriKiyosue, H. Shimizu, M. Uesugi, Y. Ishihama, M. Iwata, Y. Mizui, Nat. Chem. Biol. 2007, 3 , 570.

[8] J. Eirich, R. Orth, S. A. Sieber, J. Am. Chem. Soc. 2011, 133, 12144

[9] K. Lee, J. H. Lee, S. K. Boovanahalli, Y. Jin, M Lee, X. Jin, J. H. Kim, Y. S. Hong, J. J. Lee, J. Med. Chem. 2007, 50, 1675.

[10] K. Lee, H. S. Ban, R. Naik, Y. S. Hong, S. Son, B. K. Kim, Y. Xia, K. B. Song, H. S. Lee, M. Won, Angew. Chem. Int. Ed. 2013, 52, 10286.

[11] M. Koh, J. Park, J. Y. Koo, D. Lim, M. Y. Cha, A. Jo, J. H. Choi, S. B. Park, Angew. Chem. Int Ed. 2014, 53, 5102 .

[12] E. Nakamaru-Ogiso, B. B. Seo, T. Yagi, A. Matsuno-Yagi, FEBS Lett. 2003, 549, 43.

[13] M. Murai, S. Murakami, T. Ito, H. Miyoshi, Biochemistry 2015, 54, 2739.

[14] T. Peng, X. Yuan, H. C. Hang, Curr. Opin. Chem. Biol. 2014, 21, 144

[15] X. Liu, T. Dong, Y. Zhou, N. Huang, X. Lei, Angew. Chem. Int. Ed. 2016, 55, 14330.
[16] Y. Yang, H. Song, P. R. Chen, IUBMB. life 2016, 68, 879 .

[17] a) J. W. Chin, T. A. Cropp, J. C. Anderson, M. Mukherji, Z. Zhang, P. G. Schultz, Science 2003, 301, 964; b) Q. Wang, A. R. Parrish, L. Wang, Chem. Biol. 2009, 16, 323; c) J. T. Ngo, D. A. Tirrell, Acc. Chem. Res. 2011, 44, 677.

[18] J. C. Kauer, S. Erickson-Viitanen, J. Henry R. Wolfe, W. DeGrado, J. Biol. Chem. 1986, 261, 10695.

[19] a) J. W. Chin, A. B. Martin, D. S. King, L. Wang, P. G. Schultz, Proc. Natl. Acad. Sci. USA 2002, 99, 11020; b) J. W. Chin, P. G. Schultz, ChemBioChem 2002, 11, 1135; c) I. S. Farrell, R. Toroney, J. L. Hazen, R. A. Mehl, J. W. Chin, Nat. Methods 2005, 2, 377.

[20] J. W. Chin, S. W. Santoro, A. B. Martin, D. S. King, L. Wang, P. G. Schultz, J. Am. Chem. Soc. 2002, 124, 9026

[21] E. M. Tippmann, W. Liu, D. Summerer, A. V. Mack, P. G. Schultz, ChemBioChem 2007, 8, 2210.

[22] N. Hino, Y. Okazaki, T. Kobayashi, A. Hayashi, K. Sakamoto, S. Yokoyama, Nat. Methods $\mathbf{2 0 0 5}, 2,201$.

[23] N. Hino, M. Oyama, A. Sato, T. Mukai, F. Iraha, A. Hayashi, H. Kozuka-Hata, T. Yamamoto, S. Yokoyama, K. Sakamoto, J. Mol. Biol. 2011, 406, 343 .

[24] K. A. Daggett, T. P. Sakmar, Curr. Opin. Chem. Biol. 2011, 15, 392.

[25] A. Grunbeck, T. Huber, P. Sachdev, T. P. Sakmar, Biochemistry 2011, 50, 3411.

[26] a) S. Ye, C. Kohrer, T. Huber, M. Kazmi, P. Sachdev, E. C. Yan, A. Bhagat, U. L. RajBhandary, T. P. Sakmar, J. Biol. Chem. 2008, 283, 1525; b) A. Grunbeck, T. Huber, R. Abrol, B. Trzaskowski, W. A. Goddard, 3rd, T. P. Sakmar, ACS Chem. Biol. 2012, 7, 967.

[27] C. Koole, C. A. Reynolds, J. C. Mobarec, C. Hick, P. M. Sexton, T. P. Sakmar, J. Biol. Chem. 2017, 292, 7131

[28] a) I. Coin, M. H. Perrin, W. W. Vale, L. Wang, Angew. Chem. Int. Ed. 2011, 50, 8077; b) I. Coin, V. Katritch, T. Sun, Z. Xiang, F. Y. Siu, M. Beyermann, R. C. Stevens, L. Wang, Cell 2013 , 155,1258 .

[29] a) H. Rannversson, J. Andersen, L. Sorensen, B. Bang-Andersen, M. Park, T. Huber, T. P. Sakmar, K. Stromgaard, Nat. Commun. 2016, 7, 11261; b) H. Rannversson, J. Andersen, B. Bang-Andersen, K. Stromgaard, ACS Chem. Biol. 2017, 12, 2558.
[30] J. E. Lopez, S. E. Haynes, J. D. Majmudar, B. R Martin, C. A. Fierke, J. Am. Chem. Soc. 2017, 139,16222

[31] C. Y. Majmudar, L. W. Lee, J. K. Lancia, A. Nwokoye, Q. Wang, A. M. Wands, L. Wang, A. K. Mapp, J. Am. Chem. Soc. 2009, 131, 14240.

[32] A. Dugan, C. Y. Majmudar, R. Pricer, S. Niessen, J. K. Lancia, H. Y. Fung, B. F. Cravatt, A. K. Mapp, J. Am. Chem. Soc. 2016, 138 12629

[33] W. Wan, J. M. Tharp, W. R. Liu, Biochim. Biophys. Acta 2014, 1844, 1059.

[34] a) C. Chou, R. Uprety, L. Davis, J. W. Chin, A. Deiters, Chem. Sci. 2011, 2, 480; b) H. W. Ai, W. Shen, A. Sagi, P. R. Chen, P. G. Schultz, ChemBioChem 2011, 12, 1854

[35] a) M. Zhang, S. Lin, X. Song, J. Liu, Y. Fu, X. Ge, X. Fu, Z. Chang, P. R. Chen, Nat. Chem. Biol. 2011, 7, 671; b) S. Lin, Z. Zhang, H. Xu, L. Li, S. Chen, J. Li, Z. Hao, P. R. Chen, J. Am. Chem. Soc. 2011, 133, 20581.

[36] a) S. Zhang, D. He, Z. Lin, Y. Yang, H. Song, P. R. Chen, Acc. Chem. Res. 2017, 50, 1184; b) Y. Yang, H. Song, D. He, S. Zhang, S. Dai, X. Xie, S. Lin, Z. Hao, H. Zheng, P. R. Chen, Nat. Protoc. 2017, 12, 2147.

[37] a) S. Lin, D. He, T. Long, S. Zhang, R. Meng, P. R. Chen, J. Am. Chem. Soc. 2014, 136, 11860 b) S. Zhang, D. He, Y. Yang, S. Lin, M. Zhang, S. Dai, P. R. Chen, Proc. Natl. Acad. Sci. USA 2016, 113, 10872 .

[38] Y. Yang, H. Song, D. He, S. Zhang, S. Dai, S. Lin, R. Meng, C. Wang, P. R. Chen, Nat. Commun. 2016, 7, 12299.

[39] D. He, X. Xie, F. Yang, H. Zhang, H. Su, Y. Ge, H. Song, P. Chen, Angew. Chem. Int. Ed. 2017 $56,14521$.

[40] T. Yanagisawa, N. Hino, F. Iraha, T. Mukai, K. Sakamoto, S. Yokoyama, Mol. Biosyst. 2012, 8 , 1131.

[41] M. Suchanek, A. Radzikowska, C. Thiele, Nat. Methods 2005, 2, 261.

[42] T. Yang, X. M. Li, X. Bao, Y. M. Fung, X. D. Li, Nat. Chem. Biol. 2016, 12, 70

[43] H. Guo, J. Xu, P. Hao, K. Ding, Z. Li, Chem. Commun. 2017, 53, 9620.

[44] Y. Tian, M. P. Jacinto, Y. Zeng, Z. Yu, J. Qu, W. R. Liu, Q. Lin, J. Am. Chem. Soc. 2017, 139, 6078.

[45] Y. Tian, Q. Lin, Chem. Commun. 2018, 54, 4449.

[46] J. Park, M. Koh, J. Y. Koo, S. Lee, S. B. Park, ACS Chem. Biol. 2016, 11, 44 